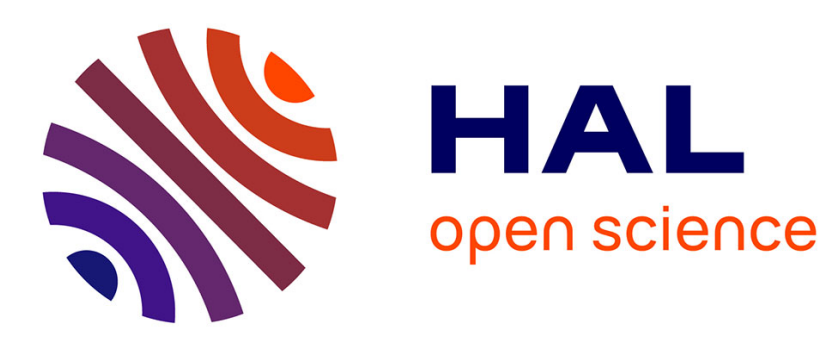

\title{
Global solutions to a degenerate solutal phase-field model for the solidification of binary alloy
}

\author{
Jean-François Scheid
}

\section{To cite this version:}

Jean-François Scheid. Global solutions to a degenerate solutal phase-field model for the solidification of binary alloy. Nonlinear Analysis: Real World Applications, 2004, 5 (1), pp.207-217. 10.1016/S14681218(03)00034-8 . hal-00141798

\section{HAL Id: hal-00141798 \\ https://hal.science/hal-00141798}

Submitted on 20 Jan 2021

HAL is a multi-disciplinary open access archive for the deposit and dissemination of scientific research documents, whether they are published or not. The documents may come from teaching and research institutions in France or abroad, or from public or private research centers.
L'archive ouverte pluridisciplinaire HAL, est destinée au dépôt et à la diffusion de documents scientifiques de niveau recherche, publiés ou non, émanant des établissements d'enseignement et de recherche français ou étrangers, des laboratoires publics ou privés. 


\title{
Global solutions to a degenerate solutal phase-field model for the solidification of binary alloy
}

\author{
J.-F. Scheid
}

\begin{abstract}
We consider a degenerate solutal phase-field model for the solidification of a binary alloy. This model is concerned with the evolution of the phase-field variable together with the relative concentration of the alloy for which the equation may degenerate. The existence of global weak solutions is proved for the degenerate case with a loss of regularity for the concentration in comparison with the non-degenerate case.
\end{abstract}

Key words : Phase-field model, degenerate parabolic systems, Faedo-Galerkin method.

\section{Introduction}

We investigate the existence of global weak solutions for the solutal and isothermal phase-field model of a binary alloy derived by Warren-Boettinger [8] in the case of a degenerate equation for the concentration. This degeneracy arises when the diffusion coefficient vanishes in the solid phase. This work is mainly based on a previous study done in [5] for the non-degenerate case. We also refer to [3] where a degenerate case is considered for a different phase-field model but whose part of this study has been inspired. In this paper, we are interested in a phase-field model describing the time evolution of an order parameter $\phi$ and the relative concentration $c$ of the alloy. The order parameter $\phi$ accounts for the solidification state of the alloy and is equal to 0 in the solid phase and equals 1 in the liquid phase. The phase-field model is given by the following equations (we refer to [2],[5] for a brief description and to $[8]$ for a full derivation) :

$$
(P) \begin{cases}\frac{\partial \phi}{\partial t}=\varepsilon^{2} \Delta \phi+F_{1}(\phi)+c F_{2}(\phi) & \text { in } \Omega \times(0,+\infty), \\ \frac{\partial c}{\partial t}=\operatorname{div}\left(D_{1}(\phi)\left(\nabla c+D_{2}(c, \phi) \nabla \phi\right)\right) & \text { in } \Omega \times(0,+\infty), \\ \frac{\partial \phi}{\partial n}=\frac{\partial c}{\partial n}=0 & \text { on } \partial \Omega \times(0,+\infty), \\ \phi(0)=\phi_{0}, \quad c(0)=c_{0} & \text { in } \Omega,\end{cases}
$$

where $\Omega$ is a open subset of $\mathbb{R}^{d}(d \leq 3)$ with a smooth boundary $\partial \Omega$ with unit normal $n$, and $\varepsilon$ is a constant. Note that, unlike the problem states in [5], the dependency of the coefficient in front of $\nabla \phi$, with the coefficient $D_{1}(\phi)$ is here explicitly pointed out. Indeed this will be a key ingredient for the analysis carried out through this paper.

Now let us precise the assumptions made on the non-linear terms in $(P)$. In this work, we assume that :

(H1) $\quad F_{1}, F_{2} \in C(\mathbb{R})$ are Lipschitz and bounded functions.

(H2) $\quad D_{1} \in C(\mathbb{R})$ is a Lipschitz non-negative and bounded function with

$$
0 \leq D_{1}(r) \leq D_{l}, \quad \forall r \in \mathbb{R} .
$$

(H3) $\quad D_{2} \in C(\mathbb{R} \times \mathbb{R})$ is a Lipschitz and bounded function.

Note that the degeneracy of the equation for $c$ is allowed through assumption (H2). A typical situation is given when $D_{1}(\phi)$ is equal to zero for $\phi=0$ i.e. $D_{1}(0)=D_{s}=0$, corresponding to the vanishing of the diffusion coefficient in the solid case. 
In addition, we also make further assumptions on the behaviour of the non-linear terms :

(H4) $\quad F_{1} \equiv F_{2} \equiv 0$ in $(-\infty, 0] \cup[1,+\infty)$.

(H5) $\quad D_{2}(., r) \equiv 0$ in $(-\infty, 0] \cup[1,+\infty)$ and for all $r \in \mathbb{R}$.

These assumptions lead to a maximum principle as we shall see later.

Throughout this article, we note $V=H^{1}(\Omega)$ and $V^{\prime}$ its dual space. The duality product between $V^{\prime}$ and $V$ is denoted by $<.,>_{V^{\prime}, V}$. Finally for $T>0$, we put $Q_{T}=\Omega \times(0, T)$.

We then state the main result about the global existence of weak solutions of Problem $(P)$ under the previous assumptions.

Theorem 1 Let $\left(\phi_{0}, c_{0}\right) \in H^{1}(\Omega) \times L^{2}(\Omega)$ such that $\phi_{0}, c_{0} \in[0,1]$ a.e. in $\Omega$. Then, under assumptions (H1) - (H5) and for any $T>0$, there exists $(\phi, c, J)$ satisfying

$$
\begin{aligned}
& \phi \in L^{2}\left(0, T ; H^{2}(\Omega)\right) \cap H^{1}\left(0, T ; L^{2}(\Omega)\right), \quad \phi(0)=\phi_{0}, \\
& c \in L^{\infty}\left(0, T ; L^{2}(\Omega)\right) \cap H^{1}\left(0, T ; V^{\prime}\right), \quad c(0)=c_{0}, \\
& J \in L^{2}\left(Q_{T}\right)^{2}, \quad J=\nabla\left(D_{1}(\phi) c\right)-c \nabla D_{1}(\phi),
\end{aligned}
$$

such that $\phi, c \in[0,1]$ a.e. in $Q_{T}$ and

$$
\begin{aligned}
& \frac{\partial \phi}{\partial t}=\varepsilon^{2} \Delta \phi+F_{1}(\phi)+c F_{2}(\phi) \quad \text { a.e. in } \Omega \times(0, T), \\
& \frac{\partial \phi}{\partial n}=0 \quad \text { a.e. on } \partial \Omega \times(0, T), \\
& <\frac{\partial c}{\partial t}, v>_{V^{\prime}, V}+\int_{\Omega}\left(J+D_{1}(\phi) D_{2}(c, \phi) \nabla \phi\right) \cdot \nabla v d x=0, \\
& \quad \text { for all } v \in H^{1}(\Omega) \text {, a.e. in }(0, T) .
\end{aligned}
$$

The proof is based on a regularization procedure of the degenerate coefficient $D_{1}$ and passage to the limit.

\section{A regularized problem}

Following [3], we regularize the coefficient $D_{1}$ by introducing a continuous function $l: \mathbb{R}^{+} \rightarrow \mathbb{R}$ such that $l(0)=0$ and $l(\lambda)>0$ for all $\lambda>0$. Then we put

$$
D_{1}^{\lambda}=D_{1}+l(\lambda)
$$

For a given $\lambda>0$, we consider the problem $\left(P^{\lambda}\right)$ obtained by putting the coefficient $D_{1}^{\lambda}$ in place of $D_{1}$ in the front of $\nabla c$ in $(P)$. In that way, since $D_{1}$ satisfies (H2), the regularized coefficient $D_{1}^{\lambda}$ satisfies for all $\lambda \in(0,1]$ the following property :

$$
\begin{array}{ll}
\text { (H2') } & D_{1}^{\lambda} \text { is a Lipschitz and bounded function such that } \\
& 0<l(\lambda) \leq D_{1}^{\lambda}(r) \leq L_{l}, \text { for all } r \in \mathbb{R} .
\end{array}
$$

Then an existence result holds for the problem $\left(P^{\lambda}\right)$ that can be read as follows (see [5], Th.1) :

Let $\left(\phi_{0}, c_{0}\right) \in H^{1}(\Omega) \times L^{2}(\Omega)$ and $T>0$. Then under assumptions (H1)-(H3), for any $\lambda \in(0,1]$ there exists $\left(\phi^{\lambda}, c^{\lambda}\right)$ satisfying

$$
\begin{aligned}
& \phi^{\lambda} \in L^{2}\left(0, T ; H^{2}(\Omega)\right) \cap H^{1}\left(0, T ; L^{2}(\Omega)\right), \quad \phi^{\lambda}(0)=\phi_{0}, \\
& c^{\lambda} \in L^{2}\left(0, T ; H^{1}(\Omega)\right) \cap H^{1}\left(0, T ; V^{\prime}\right), \quad c^{\lambda}(0)=c_{0}
\end{aligned}
$$


such that

$$
\begin{aligned}
& \frac{\partial \phi^{\lambda}}{\partial t}=\varepsilon^{2} \Delta \phi^{\lambda}+F_{1}\left(\phi^{\lambda}\right)+c F_{2}\left(\phi^{\lambda}\right) \quad \text { a.e. in } Q_{T}, \\
& \frac{\partial \phi^{\lambda}}{\partial n}=0 \quad \text { a.e. on } \partial \Omega \times(0, T), \\
& \quad<\frac{\partial c^{\lambda}}{\partial t}, v>_{V^{\prime}, V}+\int_{\Omega}\left(D_{1}^{\lambda}\left(\phi^{\lambda}\right) \nabla c^{\lambda}+D_{1}\left(\phi^{\lambda}\right) D_{2}\left(c^{\lambda}, \phi^{\lambda}\right) \nabla \phi^{\lambda}\right) \cdot \nabla v=0, \\
& \quad \text { for all } v \in H^{1}(\Omega), \text { a.e. in }(0, T) .
\end{aligned}
$$

In addition, if we assume (H4) - (H5) then a maximum principle holds (see [5], Th.3) : If $\phi_{0}, c_{0} \in[0,1]$ a.e. in $\Omega$, then for all $t \in[0, T]$,

$$
\phi^{\lambda}(t), c^{\lambda}(t) \in[0,1], \quad \text { a.e. in } \Omega .
$$

Remark : Since $\phi^{\lambda}$ and $c^{\lambda}$ satisfy the regularity mentioned in (5) and (6), we infer that $\phi^{\lambda} \in$ $C\left([0, T] ; H^{1}(\Omega)\right)$ and $c^{\lambda} \in C\left([0, T] ; L^{2}(\Omega)\right)$ and thus initial conditions in (5) and (6) make sense.

\section{Uniform estimates for the regularized problem}

We want to pass to the limit with $\lambda \rightarrow 0$ in (7), (8) and (9). To this end, we need a priori estimates for $\left(\phi^{\lambda}, c^{\lambda}\right)$ which are uniform with respect to $\lambda$. From now, we denote by $C_{T}$ a positive constant depending on $T,|\Omega|, \varepsilon,\left\|F_{1}\right\|_{L^{\infty}(\mathbb{R})},\left\|F_{2}\right\|_{L^{\infty}(\mathbb{R})},\left\|D_{1}\right\|_{W^{1, \infty}(\mathbb{R})}, L_{l},\left\|D_{2}\right\|_{L^{\infty}(\mathbb{R} \times \mathbb{R})}$ and $\left\|\phi_{0}\right\|_{H^{1}(\Omega)},\left\|c_{0}\right\|_{L^{2}(\Omega)}$ but independent of $\lambda$.

Lemma 1 There exists a positive constant $C_{T}$ such that for all $\lambda \in(0,1]$, the following estimates hold :

$$
\begin{array}{ll}
\text { i) } & \left\|\phi^{\lambda}\right\|_{L^{\infty}\left(0, T ; H^{1}(\Omega)\right)}+\left\|\phi_{t}^{\lambda}\right\|_{L^{2}\left(Q_{T}\right)}+\left\|c^{\lambda}\right\|_{L^{\infty}\left(0, T ; L^{2}(\Omega)\right)}+\int_{Q_{T}} D_{1}^{\lambda}\left(\phi^{\lambda}\right)\left|\nabla c^{\lambda}\right|^{2} d x d t \leq C_{T} \\
\text { ii) } & \left\|\phi^{\lambda}\right\|_{L^{2}\left(0, T ; H^{2}(\Omega)\right)} \leq C_{T} \\
\text { iii }) & \left\|c_{t}^{\lambda}\right\|_{L^{2}\left(0, T ; V^{\prime}\right)} \leq C_{T}
\end{array}
$$

Proof of Lemma 1 :

i) We take $c^{\lambda}$ as a test function in (9). Integrating over $(0, t)$ for $t \in[0, T]$, we obtain

$$
\frac{1}{2}\left\|c^{\lambda}(t)\right\|_{L^{2}(\Omega)}^{2}+\int_{0}^{t} \int_{\Omega} D_{1}^{\lambda}\left(\phi^{\lambda}\right)\left|\nabla c^{\lambda}\right|^{2}=\frac{1}{2}\left\|c_{0}\right\|_{L^{2}(\Omega)}^{2}-\int_{0}^{t} \int_{\Omega} D_{1}\left(\phi^{\lambda}\right) D_{2}\left(c^{\lambda}, \phi^{\lambda}\right) \nabla \phi^{\lambda} \cdot \nabla c^{\lambda} .
$$

Using the boundedness of $D_{2}$, the positivity of $D_{1}$ in assumptions (H2), (H3) and the definition (4) of $D_{1}^{\lambda}$ together with Cauchy-Schwarz and Young inequalities, we infer that, for all $t \in[0, T]$,

$$
\left|\int_{0}^{t} \int_{\Omega} D_{1}\left(\phi^{\lambda}\right) D_{2}\left(c^{\lambda}, \phi^{\lambda}\right) \nabla \phi^{\lambda} \cdot \nabla c^{\lambda}\right| \leq \frac{1}{2} \int_{0}^{t} \int_{\Omega} D_{1}^{\lambda}\left(\phi^{\lambda}\right)\left|\nabla c^{\lambda}\right|^{2}+\frac{C_{T}}{2} \int_{0}^{t} \int_{\Omega}\left|\nabla \phi^{\lambda}\right|^{2} .
$$

Then combining (11) with (12), we deduce that there exists a positive constant $C_{1}$ independent of $\lambda$ such that, for all $t \in[0, T]$,

$$
\left\|c^{\lambda}(t)\right\|_{L^{2}(\Omega)}^{2}+\int_{0}^{t} \int_{\Omega} D_{1}^{\lambda}\left(\phi^{\lambda}\right)\left|\nabla c^{\lambda}\right|^{2} \leq C_{1}\left(1+\int_{0}^{t} \int_{\Omega}\left|\nabla \phi^{\lambda}\right|^{2}\right) .
$$

Now we take the $L^{2}$-scalar product of (7) with $\phi_{t}^{\lambda}+\phi^{\lambda}$ and we integrate over $(0, t)$ for $t \in[0, T]$. After integration by parts we obtain, for all $t \in[0, T]$,

$$
\begin{gathered}
\frac{1}{2}\left(\left\|\phi^{\lambda}(t)\right\|_{L^{2}(\Omega)}^{2}+\varepsilon^{2}\left\|\nabla \phi^{\lambda}(t)\right\|_{L^{2}(\Omega)}^{2}\right)+\int_{0}^{t}\left(\left\|\phi_{t}^{\lambda}\right\|_{L^{2}(\Omega)}^{2}+\varepsilon^{2}\left\|\nabla \phi^{\lambda}\right\|_{L^{2}(\Omega)}^{2}\right) \\
=\frac{1}{2}\left(\left\|\phi_{0}\right\|_{L^{2}(\Omega)}^{2}+\varepsilon^{2}\left\|\nabla \phi_{0}\right\|_{L^{2}(\Omega)}^{2}\right) \\
\quad+\int_{0}^{t} \int_{\Omega} F_{1}\left(\phi^{\lambda}\right)\left(\phi_{t}^{\lambda}+\phi^{\lambda}\right)+\int_{0}^{t} \int_{\Omega} c^{\lambda} F_{2}\left(\phi^{\lambda}\right)\left(\phi_{t}^{\lambda}+\phi^{\lambda}\right) .
\end{gathered}
$$


Then we use Cauchy-Schwarz and Young inequalities with (H1) to estimate the right-hand term in (14). This leads to the existence of a positive constant $C_{2}$ independent of $\lambda$ such that

$$
\begin{aligned}
\left\|\phi^{\lambda}(t)\right\|_{L^{2}(\Omega)}^{2}+\varepsilon^{2}\left\|\nabla \phi^{\lambda}(t)\right\|_{L^{2}(\Omega)}^{2}+\int_{0}^{t} & \left(\left\|\phi_{t}^{\lambda}\right\|_{L^{2}(\Omega)}^{2}+\varepsilon^{2}\left\|\nabla \phi^{\lambda}\right\|_{L^{2}(\Omega)}^{2}\right) \\
& \leq C_{2}\left(1+\int_{0}^{t}\left\|\phi^{\lambda}\right\|_{L^{2}(\Omega)}^{2}+\int_{0}^{t}\left\|c^{\lambda}\right\|_{L^{2}(\Omega)}^{2}\right)
\end{aligned}
$$

Now we multiply estimate (13) by a number $\delta>0$ that will be choosen later and adding the result to estimate (15), we get, for all $t \in[0, T]$,

$$
\begin{aligned}
& \left\|\phi^{\lambda}(t)\right\|_{L^{2}(\Omega)}^{2}+\varepsilon^{2}\left\|\nabla \phi^{\lambda}(t)\right\|_{L^{2}(\Omega)}^{2}+\delta\left\|c^{\lambda}(t)\right\|^{2}+\int_{0}^{t}\left\|\phi_{t}^{\lambda}\right\|_{L^{2}(\Omega)}^{2}+\varepsilon^{2} \int_{0}^{t}\left\|\nabla \phi^{\lambda}\right\|_{L^{2}(\Omega)}^{2} \\
& +\delta \int_{0}^{t} \int_{\Omega} D_{1}^{\lambda}\left(\phi^{\lambda}\right)\left|\nabla c^{\lambda}\right|^{2} \leq \delta C_{1} \int_{0}^{t}\left\|\nabla \phi^{\lambda}\right\|_{L^{2}(\Omega)}^{2}+C_{T}\left(1+\int_{0}^{t}\left\|\phi^{\lambda}\right\|_{L^{2}(\Omega)}^{2}+\int_{0}^{t}\left\|c^{\lambda}\right\|_{L^{2}(\Omega)}^{2}\right) .
\end{aligned}
$$

We choose $\delta>0$ such that $\delta C_{1}=\varepsilon^{2} / 2$ and thus we obtain for all $t \in[0, T]$,

$$
\begin{aligned}
\left\|\phi^{\lambda}(t)\right\|_{L^{2}(\Omega)}^{2} & +\varepsilon^{2}\left\|\nabla \phi^{\lambda}(t)\right\|_{L^{2}(\Omega)}^{2}+\delta\left\|c^{\lambda}(t)\right\|^{2}+\int_{0}^{t}\left\|\phi_{t}^{\lambda}\right\|_{L^{2}(\Omega)}^{2}+\frac{\varepsilon^{2}}{2} \int_{0}^{t}\left\|\nabla \phi^{\lambda}\right\|_{L^{2}(\Omega)}^{2} \\
& +\delta \int_{0}^{t} \int_{\Omega} D_{1}^{\lambda}\left(\phi^{\lambda}\right)\left|\nabla c^{\lambda}\right|^{2} \leq C_{T}\left(1+\int_{0}^{t}\left\|\phi^{\lambda}\right\|_{L^{2}(\Omega)}^{2}+\int_{0}^{t}\left\|c^{\lambda}\right\|_{L^{2}(\Omega)}^{2}\right) .
\end{aligned}
$$

Applying Gronwall's lemma, we conclude that

$$
\left\|\phi^{\lambda}(t)\right\|_{L^{2}(\Omega)}^{2}+\varepsilon^{2}\left\|\nabla \phi^{\lambda}(t)\right\|_{L^{2}(\Omega)}^{2}+\delta\left\|c^{\lambda}(t)\right\|^{2} \leq C_{T}, \quad \text { for all } t \in[0, T]
$$

and thus

$$
\left\|\phi^{\lambda}\right\|_{L^{\infty}\left(0, T ; H^{1}(\Omega)\right)}+\left\|c^{\lambda}\right\|_{L^{\infty}\left(0, T ; L^{2}(\Omega)\right)} \leq C_{T} .
$$

Finally, estimate (16) with (17) leads to the desired estimate $i$ ) of Lemma 1.

ii) We take the $L^{2}$-scalar product of equation (7) with $-\Delta \phi^{\lambda}$ and we integrate over $(0, t)$ for $t \in[0, T]$. After integration by parts and the use of Cauchy-Schwarz and Young inequalities with (H1), we have, for all $t \in[0, T]$,

$$
\left\|\nabla \phi^{\lambda}(t)\right\|_{L^{2}(\Omega)}^{2}+\varepsilon^{2} \int_{0}^{t}\left\|\Delta \phi^{\lambda}\right\|_{L^{2}(\Omega)}^{2} \leq C_{T}\left(1+\int_{0}^{t}\left\|c^{\lambda}\right\|_{L^{2}(\Omega)}^{2}\right) .
$$

We deduce using estimate $i$ ) of Lemma 1 that

$$
\left\|\Delta \phi^{\lambda}\right\|_{L^{2}\left(Q_{T}\right)} \leq C_{T}
$$

We conclude using well-known elliptic results that estimate $i$ ) holds.

iii) From equation (9) with (H1), (H2), (H3), it is straightforward to get

$$
\left\|c_{t}^{\lambda}\right\|_{L^{2}\left(0, T ; V^{\prime}\right)} \leq C_{T}\left(\int_{Q_{T}} D_{1}^{\lambda}\left(\phi^{\lambda}\right)\left|\nabla c^{\lambda}\right|^{2}+\left\|\phi^{\lambda}\right\|_{L^{\infty}\left(0, T ; H^{1}(\Omega)\right)}^{2}\right) .
$$

Then we conclude using estimate $i$ ), that estimate iii) holds.

\section{Passage to the limit with $\lambda \rightarrow 0$.}

We want now to pass to the limit in (7), (8) and (9) as $\lambda$ tends to 0. From Lemma 1, we know that $\left\{\phi^{\lambda}\right\}$ is bounded in

$$
W_{1}=\left\{v \in L^{2}\left(0, T ; H^{2}(\Omega)\right), v_{t} \in L^{2}\left(0, T ; L^{2}(\Omega)\right)\right\}
$$

and in

$$
W_{2}=\left\{v \in L^{\infty}\left(0, T ; H^{1}(\Omega)\right), v_{t} \in L^{2}\left(0, T ; L^{2}(\Omega)\right)\right\}
$$


Since $W_{1}$ and $W_{2}$ are compactly embedded respectively into $L^{2}\left(0, T ; H^{1}(\Omega)\right)$ and into $C\left([0, T] ; L^{2}(\Omega)\right)$ (see [6], Cor. 4), then there exists a function

$$
\phi \in L^{2}\left(0, T ; H^{2}(\Omega)\right) \cap H^{1}\left(0, T ; L^{2}(\Omega)\right) \cap L^{\infty}\left(0, T ; H^{1}(\Omega)\right)
$$

and a subsequence still denoted by $\phi^{\lambda}$, such that as $\lambda$ tends to 0 ,

$$
\begin{aligned}
& \phi^{\lambda} \rightarrow \phi \text { in } L^{2}\left(0, T ; H^{2}(\Omega)\right) \text { weak, } \\
& \phi_{t}^{\lambda} \rightarrow \phi_{t} \text { in } L^{2}\left(Q_{T}\right) \text { weak, } \\
& \phi^{\lambda} \rightarrow \phi \text { in } L^{2}\left(0, T ; H^{1}(\Omega)\right) \text { and in } C\left([0, T] ; L^{2}(\Omega)\right) \text { strong. }
\end{aligned}
$$

On the other hand, we know that $\left\{c^{\lambda}\right\}$ is bounded in

$$
W_{3}=\left\{v \in L^{\infty}\left(0, T ; L^{2}(\Omega)\right), v_{t} \in L^{2}\left(0, T ; V^{\prime}\right)\right\}
$$

and in $L^{\infty}\left(Q_{T}\right)$ due to the maximum principle. Since $W_{3}$ is compactly embedded in $C\left([0, T] ; V^{\prime}\right)$ (see $[6])$, there exists a function

$$
c \in L^{\infty}\left(0, T ; L^{2}(\Omega)\right) \cap H^{1}\left(0, T ; V^{\prime}\right) \cap L^{\infty}\left(Q_{T}\right)
$$

and a subsequence still denoted by $c^{\lambda}$, such that as $\lambda$ tends to 0 ,

$$
\begin{aligned}
& c^{\lambda} \rightarrow c \text { in } L^{2}\left(Q_{T}\right) \text { weak, and in } L^{\infty}\left(Q_{T}\right) \text { weak-*, } \\
& c_{t}^{\lambda} \rightarrow c_{t} \text { in } L^{2}\left(0, T ; V^{\prime}\right) \text { weak, } \\
& c^{\lambda} \rightarrow c \text { in } C\left([0, T] ; V^{\prime}\right) \text { strong. }
\end{aligned}
$$

Finally, from Lemma 1-i) and property (H2'), we infer that $D_{1}^{\lambda}\left(\phi^{\lambda}\right) \nabla c^{\lambda}$ is uniformly bounded in $L^{2}\left(Q_{T}\right)^{2}$ and thus there exists a function

$$
J \in L^{2}\left(Q_{T}\right)^{2}
$$

such that

$$
D_{1}^{\lambda}\left(\phi^{\lambda}\right) \nabla c^{\lambda} \rightarrow J \text { in } L^{2}\left(Q_{T}\right)^{2} \text { weak }
$$

We now obtain a first convergence result for the nonlinear terms.

Lemma 2 As $\lambda \rightarrow 0$, we have

$$
\begin{array}{lll}
\text { i) } & F_{i}\left(\phi^{\lambda}\right) \rightarrow F_{i}(\phi) \quad i=1,2 & \text { in } L^{p}\left(Q_{T}\right), \forall p \in[1,+\infty), \\
\text { ii) } & c^{\lambda} F_{2}\left(\phi^{\lambda}\right) \rightarrow c F_{2}(\phi) & \text { in } L^{2}\left(Q_{T}\right) \text { weak } \\
\text { iii) } & D_{1}\left(\phi^{\lambda}\right) \rightarrow D_{1}(\phi) & \text { in } L^{2}\left(0, T ; H^{1}(\Omega)\right) \\
\text { iv) } & c^{\lambda} D_{1}\left(\phi^{\lambda}\right) \rightarrow c D_{1}(\phi) & \text { in } L^{2}\left(Q_{T}\right) \text { weak } \\
\text { v) } & c^{\lambda} \nabla D_{1}\left(\phi^{\lambda}\right) \rightarrow c \nabla D_{1}(\phi) & \text { in } L^{2}\left(Q_{T}\right)^{2} \text { weak. }
\end{array}
$$

\section{Proof of Lemma 2:}

i) From (23), we have that $\phi^{\lambda} \rightarrow \phi$ a.e. in $Q_{T}$ and since $F_{i}$ are Lipschitz functions, we conclude by Lebesgue dominated convergence theorem that $F_{i}\left(\phi^{\lambda}\right) \rightarrow F_{i}(\phi)$ in $L^{p}\left(Q_{T}\right)$ for all $p \in[1,+\infty)$.

ii) For $v \in L^{2}\left(Q_{T}\right)$, we have

$$
\int_{Q_{T}}\left(c^{\lambda} F_{2}\left(\phi^{\lambda}\right)-c F_{2}(\phi)\right) v=\int_{Q_{T}}\left(c^{\lambda}-c\right) F_{2}(\phi) v+\int_{Q_{T}} c^{\lambda}\left(F_{2}\left(\phi^{\lambda}\right)-F_{2}(\phi)\right) v .
$$

The first term on the right hand side tends to 0 since $F_{2}(\phi) v$ belongs to $L^{2}\left(Q_{T}\right)$ and due to (24). The second term also tends to 0 since $0 \leq c^{\lambda} \leq 1$ a.e. in $Q_{T}$ and thanks to (29). 
iii) $D_{1}$ is Lipschitz and thanks to (23), we conclude (see [1], Th. 16.7) that $D_{1}\left(\phi^{\lambda}\right) \rightarrow D_{1}(\phi)$ in $L^{2}\left(0, T ; H^{1}(\Omega)\right)$.

iv) The proof is the same as for $i i)$.

$v)$ Let $v \in L^{2}\left(Q_{T}\right)^{2}$. We have

$$
\int_{Q_{T}}\left(c^{\lambda} \nabla D_{1}\left(\phi^{\lambda}\right)-c \nabla D_{1}(\phi)\right) \cdot v=\int_{Q_{T}}\left(c^{\lambda}-c\right) \nabla D_{1}(\phi) \cdot v+\int_{Q_{T}} c^{\lambda}\left(\nabla D_{1}\left(\phi^{\lambda}\right)-\nabla D_{1}(\phi)\right) \cdot v .
$$

The first term on the right hand side tends to 0 since $D_{1} \in W^{1, \infty}(\mathbb{R})$ and $\nabla D_{1}(\phi) \cdot v=D_{1}^{\prime}(\phi) \nabla \phi \cdot v \in$ $L^{2}\left(Q_{T}\right)$ and due to (24). The second term tends also to 0 since iii) holds and $0 \leq c^{\lambda} \leq 1$ a.e. on $Q_{T}$. Hence $c^{\lambda} \nabla D_{1}\left(\phi^{\lambda}\right) \rightarrow c \nabla D_{1}(\phi)$ in $L^{2}\left(Q_{T}\right)^{2}$ weak.

We can now identify the function $J$ as it is done in [3].

Lemma 3 As $\lambda \rightarrow 0$, we have

$$
D_{1}^{\lambda}\left(\phi^{\lambda}\right) \nabla c^{\lambda} \rightarrow J \text { in } L^{2}\left(Q_{T}\right)^{2} \text { weak. }
$$

with

$$
J=\nabla\left(D_{1}(\phi) c\right)-c \nabla D_{1}(\phi)
$$

Proof of Lemma 3: The proof is similar to [3]. Since $D_{1}\left(\phi^{\lambda}\right) \in L^{2}\left(0, T ; H^{1}(\Omega)\right)$ and $c^{\lambda} \in L^{\infty}\left(0, T ; H^{1}(\Omega)\right)$, we first infer that $D_{1}\left(\phi^{\lambda}\right) c^{\lambda} \in L^{2}\left(0, T ; W^{1,3 / 2}(\Omega)\right)$ (see for instance [7]) and

$$
\nabla\left(D_{1}\left(\phi^{\lambda}\right) c^{\lambda}\right)=D_{1}\left(\phi^{\lambda}\right) \nabla c^{\lambda}+c^{\lambda} \nabla D_{1}\left(\phi^{\lambda}\right) \quad \text { a.e. in } Q_{T} .
$$

Furthermore, since $0 \leq c^{\lambda} \leq 1$ a.e. in $Q_{T}$, we infer that $D_{1}\left(\phi^{\lambda}\right) c^{\lambda} \in L^{2}\left(0, T ; H^{1}(\Omega)\right)$.

Now, from Lemma 2-iv), we deduce that

$$
\nabla\left(D_{1}\left(\phi^{\lambda}\right) c^{\lambda}\right) \rightarrow \nabla\left(D_{1}(\phi) c\right) \text { in } \mathcal{D}^{\prime}\left(Q_{T}\right)
$$

and from Lemma $2-v$ ) that

$$
c^{\lambda} \nabla D_{1}\left(\phi^{\lambda}\right) \rightarrow c \nabla D_{1}(\phi) \text { in } \mathcal{D}^{\prime}\left(Q_{T}\right)
$$

Thus (36) leads to

$$
D_{1}\left(\phi^{\lambda}\right) \nabla c^{\lambda} \rightarrow \nabla\left(D_{1}(\phi) c\right)-c \nabla D_{1}(\phi) \text { in } \mathcal{D}^{\prime}\left(Q_{T}\right) .
$$

Moreover, recall that $D_{1}^{\lambda}=D_{1}+l(\lambda)$ and then since $D_{1}$ is nonnegative, we deduce from Lemma 1-i),

$$
l(\lambda) \int_{Q_{T}}\left|\nabla c^{\lambda}\right|^{2} \leq C_{T} .
$$

Therefore $\left\|l(\lambda) \nabla c^{\lambda}\right\|_{L^{2}\left(Q_{T}\right)}^{2} \leq C_{T} l(\lambda)$ and then $l(\lambda) \nabla c^{\lambda} \rightarrow 0$ in $L^{2}\left(Q_{T}\right)^{2}$ as $\lambda \rightarrow 0$. Hence we deduce from (37) that

$$
D_{1}^{\lambda}\left(\phi^{\lambda}\right) \nabla c^{\lambda} \rightarrow \nabla\left(D_{1}(\phi) c\right)-c \nabla D_{1}(\phi) \text { in } \mathcal{D}^{\prime}\left(Q_{T}\right) .
$$

We conclude from (28) and (38) that $J=\nabla\left(D_{1}(\phi) c\right)-c \nabla D_{1}(\phi)$.

The next Lemma states a further strong convergence result about the function product $D_{1}\left(\phi^{\lambda}\right) c^{\lambda}$ and allows us to pass to the limit in the term involving with $D_{2}$.

Lemma 4 i) There exists a positive constant $C_{T}$ such that for all $\lambda \in(0,1]$, we have

$$
\left\|D_{1}\left(\phi^{\lambda}\right) c^{\lambda}\right\|_{L^{2}\left(0, T ; H^{1}(\Omega)\right)} \leq C_{T} .
$$

ii) As $\lambda \rightarrow 0$, we have

$$
\begin{array}{ll}
\text { a) } & D_{1}\left(\phi^{\lambda}\right) c^{\lambda} \rightarrow D_{1}(\phi) c \quad \text { in } L^{2}\left(Q_{T}\right), \\
\text { b) } & D_{1}\left(\phi^{\lambda}\right) D_{2}\left(c^{\lambda}, \phi^{\lambda}\right) \nabla \phi^{\lambda} \rightarrow D_{1}(\phi) D_{2}(c, \phi) \nabla \phi \quad \text { in } L^{1}\left(Q_{T}\right)^{2} \text { weak. }
\end{array}
$$


Proof of Lemma 4 :

i) We prove that $D_{1}\left(\phi^{\lambda}\right) c^{\lambda}$ is uniformly bounded in $L^{2}\left(0, T ; H^{1}(\Omega)\right)$. From $(36)$, we deduce that

$$
\int_{Q_{T}}\left|\nabla\left(D_{1}\left(\phi^{\lambda}\right) c^{\lambda}\right)\right|^{2} \leq \int_{Q_{T}} D_{1}\left(\phi^{\lambda}\right)^{2}\left|\nabla c^{\lambda}\right|^{2}+\int_{Q_{T}}\left|c^{\lambda} D_{1}^{\prime}\left(\phi^{\lambda}\right) \nabla \phi^{\lambda}\right|^{2} .
$$

Then using Lemma 1-i) with the fact that $D_{1} \leq D_{1}^{\lambda}$ and $D_{1} \in W^{1, \infty}(\mathbb{R})$, we obtain

$$
\begin{aligned}
\int_{Q_{T}}\left|\nabla\left(D_{1}\left(\phi^{\lambda}\right) c^{\lambda}\right)\right|^{2} & \leq C_{T}\left(1+\int_{Q_{T}}\left|c^{\lambda} \nabla \phi^{\lambda}\right|^{2}\right) \\
& \leq C_{T}\left(1+\left\|\nabla \phi^{\lambda}\right\|_{L^{2}\left(Q_{T}\right)}^{2}\right) \\
& \leq C_{T},
\end{aligned}
$$

where we have also used the fact that $0 \leq c^{\lambda} \leq 1$ a.e. in $Q_{T}$ and estimate $i$ ) of Lemma 1 .

ii)-a) Since $D_{1}\left(\phi^{\lambda}(t)\right) c^{\lambda}(t)-D_{1}(\phi(t)) c(t) \in H^{1}(\Omega)$ for a.e. $t \in(0, T)$, we can apply a well-known compacteness inequality (see for instance [4], Lemma 5.1) : For all $\eta>0$, there exists a positive constant $C_{\eta}$ such that

$$
\begin{aligned}
\left\|D_{1}\left(\phi^{\lambda}(t)\right) c^{\lambda}(t)-D_{1}(\phi(t)) c(t)\right\|_{L^{2}(\Omega) \leq} & \eta\left\|D_{1}\left(\phi^{\lambda}(t)\right) c^{\lambda}(t)-D_{1}(\phi(t)) c(t)\right\|_{H^{1}(\Omega)} \\
& +C_{\eta}\left\|D_{1}\left(\phi^{\lambda}(t)\right) c^{\lambda}(t)-D_{1}(\phi(t)) c(t)\right\|_{V^{\prime}} .
\end{aligned}
$$

Then integrating over $t \in(0, T)$, we have : $\forall \eta>0$, there exists $C_{\eta}$ such that

$$
\begin{aligned}
\left\|D_{1}\left(\phi^{\lambda}\right) c^{\lambda}-D_{1}(\phi) c\right\|_{L^{2}\left(Q_{T}\right) \leq} & \eta\left\|D_{1}\left(\phi^{\lambda}\right) c^{\lambda}-D_{1}(\phi) c\right\|_{L^{2}\left(0, T ; H^{1}(\Omega)\right)} \\
& +C_{\eta}\left\|D_{1}\left(\phi^{\lambda}\right) c^{\lambda}-D_{1}(\phi) c\right\|_{L^{2}\left(0, T ; V^{\prime}\right)}
\end{aligned}
$$

Moreover, we have

$$
\left\|D_{1}\left(\phi^{\lambda}\right) c^{\lambda}-D_{1}(\phi) c\right\|_{L^{2}\left(0, T ; V^{\prime}\right)} \leq\left\|D_{1}(\phi)\left(c^{\lambda}-c\right)\right\|_{L^{2}\left(0, T ; V^{\prime}\right)}+\left\|c^{\lambda}\left(D_{1}\left(\phi^{\lambda}\right)-D_{1}(\phi)\right)\right\|_{L^{2}\left(0, T ; V^{\prime}\right)}
$$

and since $D_{1}$ is a bounded function and $0 \leq c^{\lambda} \leq 1$ a.e. in $Q_{T}$, there exists a constant $C_{1}$ independent of $\lambda$ such that

$$
\left\|D_{1}\left(\phi^{\lambda}\right) c^{\lambda}-D_{1}(\phi) c\right\|_{L^{2}\left(0, T ; V^{\prime}\right)} \leq C_{1}\left(\left\|c^{\lambda}-c\right\|_{L^{2}\left(0, T ; V^{\prime}\right)}+\left\|D_{1}\left(\phi^{\lambda}\right)-D_{1}(\phi)\right\|_{L^{2}\left(0, T ; V^{\prime}\right)}\right) .
$$

Combining (43) with (44), we obtain : $\forall \eta>0$, there exists $C_{\eta}^{\prime}$ such that

$$
\begin{aligned}
\left\|D_{1}\left(\phi^{\lambda}\right) c^{\lambda}-D_{1}(\phi) c\right\|_{L^{2}\left(Q_{T}\right) \leq} & \eta\left\|D_{1}\left(\phi^{\lambda}\right) c^{\lambda}-D_{1}(\phi) c\right\|_{L^{2}\left(0, T ; H^{1}(\Omega)\right)} \\
& +C_{\eta}^{\prime}\left(\left\|c^{\lambda}-c\right\|_{L^{2}\left(0, T ; V^{\prime}\right)}+\left\|D_{1}\left(\phi^{\lambda}\right)-D_{1}(\phi)\right\|_{L^{2}\left(0, T ; V^{\prime}\right)}\right) .
\end{aligned}
$$

Now let $\varepsilon>0$ be fixed. Thanks to (39), there exists a positive constant $C_{2}$ independent of $\lambda$ such that $\left\|D_{1}\left(\phi^{\lambda}\right) c^{\lambda}-D_{1}(\phi) c\right\|_{L^{2}\left(0, T ; H^{1}(\Omega)\right)} \leq C_{2}$ and then

$$
\left\|D_{1}\left(\phi^{\lambda}\right) c^{\lambda}-D_{1}(\phi) c\right\|_{L^{2}\left(Q_{T}\right)} \leq \frac{\varepsilon}{2}+C_{\eta}^{\prime}\left(\left\|c^{\lambda}-c\right\|_{L^{2}\left(0, T ; V^{\prime}\right)}+\left\|D_{1}\left(\phi^{\lambda}\right)-D_{1}(\phi)\right\|_{L^{2}\left(0, T ; V^{\prime}\right)}\right),
$$

if we choose $\eta$ such that $\eta C_{2} \leq \varepsilon / 2$. We conclude using the strong convergences (26) and (31) that

$$
\left\|D_{1}\left(\phi^{\lambda}\right) c^{\lambda}-D_{1}(\phi) c\right\|_{L^{2}\left(Q_{T}\right)} \leq \varepsilon \text { for } \lambda \text { small enough, }
$$

and therefore $D_{1}\left(\phi^{\lambda}\right) c^{\lambda} \rightarrow D_{1}(\phi) c$ in $L^{2}\left(Q_{T}\right)$ as $\lambda \rightarrow 0$.

ii)-b) Let $v \in L^{\infty}\left(Q_{T}\right)^{2}$ and we put

$$
\begin{aligned}
& \mathcal{A}=\int_{Q_{T}}\left(D_{1}\left(\phi^{\lambda}\right) D_{2}\left(c^{\lambda}, \phi^{\lambda}\right) \nabla \phi^{\lambda}-D_{1}(\phi) D_{2}(c, \phi) \nabla \phi\right) \cdot v \\
& =\int_{Q_{T}}^{Q_{T}} D_{1}\left(\phi^{\lambda}\right)\left(D_{2}\left(c^{\lambda}, \phi^{\lambda}\right)-D_{2}(c, \phi)\right) \nabla \phi \cdot v \\
& +\int_{Q_{T}}\left(D_{1}\left(\phi^{\lambda}\right)-D_{1}(\phi)\right) D_{2}(c, \phi) \nabla \phi \cdot v \\
& +\int_{Q_{T}}^{Q_{T}} D_{1}\left(\phi^{\lambda}\right) D_{2}\left(c^{\lambda}, \phi^{\lambda}\right)\left(\nabla \phi^{\lambda}-\nabla \phi\right) \cdot v
\end{aligned}
$$


From (23) and Lemma 2-iii), we obtain $E 2 \rightarrow 0$ and $E 3 \rightarrow 0$. Using the Lipschitz property of $D_{2}$, we get

$$
|E 1| \leq C_{T} \int_{Q_{T}} D_{1}\left(\phi^{\lambda}\right)\left(\left|c^{\lambda}-c\right|+\left|\phi^{\lambda}-\phi\right|\right)|\nabla \phi| .
$$

Since $D_{1}$ is a nonnegative function, we can write

$$
\int_{Q_{T}} D_{1}\left(\phi^{\lambda}\right)\left(\left|c^{\lambda}-c\right|+\left|\phi^{\lambda}-\phi\right|\right)|\nabla \phi|=\int_{Q_{T}}\left|D_{1}\left(\phi^{\lambda}\right) c^{\lambda}-D_{1}\left(\phi^{\lambda}\right) c\right||\nabla \phi|+\int_{Q_{T}} D_{1}\left(\phi^{\lambda}\right)\left|\phi^{\lambda}-\phi\right||\nabla \phi|
$$

and then with (45) this leads to

$$
\begin{aligned}
|E 1| \leq C_{T}\left(\int_{Q_{T}}\left|D_{1}\left(\phi^{\lambda}\right) c^{\lambda}-D_{1}(\phi) c\right||\nabla \phi|+\int_{Q_{T}}\left|D_{1}(\phi)-D_{1}\left(\phi^{\lambda}\right)\right||c||\nabla \phi|\right. & \\
& \left.+\int_{Q_{T}}\left|\phi^{\lambda}-\phi\right||\nabla \phi|\right) .
\end{aligned}
$$

From the strong convergences (40),(23) and Lemma 2 with the fact that $c \in L^{\infty}\left(Q_{T}\right)$, we conclude that $E 1 \rightarrow 0$. Thus we have proved that $\mathcal{A} \rightarrow 0$.

Remark : Without using the maximum principle i.e. the fact that $0 \leq c^{\lambda} \leq 1$ in $Q_{T}$, we can obtain weaker convergence results in Lemma 2 and Lemma 3 but which are still enough to pass to the limit in the related nonlinear terms. However, the $L^{\infty}$-bound for $c^{\lambda}$ is necessary in order to get estimate (39).

Now we are able to pass to the limit with $\lambda \rightarrow 0$ in equations (7), (8) and (9) by the use of Lemma 2,3 and 4 and we obtain equations (1), (2) and (3). In addition, from (23) and (26) we infer that $\phi(0)=\phi_{0}$ and $c(0)=c_{0}$. Finally the weak convergence (24) leads to $\|c\|_{L^{\infty}\left(Q_{T}\right)} \leq \lim \inf \left\|c^{\lambda}\right\|_{L^{\infty}\left(Q_{T}\right)}$ which can be used to prove that $0 \leq c \leq 1$ a.e. in $Q_{T}$. The same holds for the function $\phi$ and the proof of Theorem 1 is completed.

\section{References}

[1] O. Kavian, Introduction à la théorie des points critiques et applications aux problèmes elliptiques, Mathématiques et Applications 13, SMAI, Springer-Verlag, Paris 1993.

[2] D. Kessler, O. Krüger, J. Rappaz, J.-F. Scheid, 'A phase-field model for the isothermal solidification of a binary alloy', Computer Assisted Mechanics and Engineering Sciences, 7, 279-288, (2000);

[3] Ph. Laurençot, 'Weak solutions to a phase-field model with non-constant thermal conductivity', Quart. Appl. Math., 4, 739-760 (1997).

[4] J.L. Lions, Quelques méthodes de résolution des problèmes aux limites non linéaires, Dunod Gauthier-Villars, Paris, 1969.

[5] J. Rappaz, J.-F. Scheid, 'Existence of solutions to a phase-field model for the isothermal solidification process of binary alloys', Math. Meth. Appl. Sci., Vol. 23, No. 6, 2000, pp. 491-513.

[6] J. Simon, 'Compact sets in the space $L^{p}(0, T ; B)$ ', Ann. Mat. Pura Appl., 146, 65-96 (1987).

[7] T. Valent, 'A property of multiplication in Sobolev spaces', Rend. Sem. Mat. Univ. Padova, Vol. 74, 63-73 (1985).

[8] J.A. Warren, W.J. Boettinger, 'Prediction of dendritic growth and microsegregation patterns in a binary alloy using the phase-field model', Acta metall. mater., 43 (2), 689-703 (1995). 